\title{
Optogenetic Manipulation of Neuronal Activity to Modulate Behavior in Freely Moving Mice
}

\author{
Laura Berg ${ }^{1,2}$, Jill Gerdey ${ }^{1,2}$, Olivia A. Masseck $k^{1,2}$ \\ ${ }^{1}$ Biology and Chemistry, Synthetic Biology, University of Bremen ${ }^{2}$ Advanced Fluorescence Microscopy, Faculty of Biology and Biotechnology, Ruhr- \\ Universität Bochum
}

\section{Corresponding Author}

Olivia A. Masseck

masseck@uni-bremen.de

\section{Citation}

Berg, L., Gerdey, J.,

Masseck, O.A. Optogenetic Manipulation of Neuronal Activity to Modulate Behavior in Freely Moving Mice. J. Vis. Exp. (164), e61023, doi:10.3791/61023 (2020).

\section{Date Published}

October 27, 2020

\section{DOI}

$10.3791 / 61023$

URL

jove.com/video/61023

\section{Abstract}

Optogenetic modulation of neuronal circuits in freely moving mice affects acute and long-term behavior. This method is able to perform manipulations of single neurons and region-specific transmitter release, up to whole neuronal circuitries in the central nervous system, and allows the direct measurement of behavioral outcomes. Neurons express optogenetic tools via an injection of viral vectors carrying the DNA of choice, such as Channelrhodopsin2 (ChR2). Light is brought into specific brain regions via chronic optical implants that terminate directly above the target region. After two weeks of recovery and proper tool-expression, mice can be repeatedly used for behavioral tests with optogenetic stimulation of the neurons of interest.

Optogenetic modulation has a high temporal and spatial resolution that can be accomplished with high cell specificity, compared to the commonly used methods such as chemical or electrical stimulation. The light does not harm neuronal tissue and can therefore be used for long-term experiments as well as for multiple behavioral experiments in one mouse. The possibilities of optogenetic tools are nearly unlimited and enable the activation or silencing of whole neurons, or even the manipulation of a specific receptor type by light.

The results of such behavioral experiments with integrated optogenetic stimulation directly visualizes changes in behavior caused by the manipulation. The behavior of the same animal without light stimulation as a baseline is a good control for induced changes. This allows a detailed overview of neuronal types or neurotransmitter systems involved in specific behaviors, such as anxiety. The plasticity of neuronal networks can also be investigated in great detail through long-term stimulation or behavioral observations after optical stimulation. Optogenetics will help to enlighten neuronal signaling in several kinds of neurological diseases. 


\section{Introduction}

The modulation of neuronal circuits in the central nervous system and their behavioral outcomes are important for understanding how the brain works, especially in psychiatric diseases and cognitive tasks such as learning and memory. With optogenetics, single cells or cell populations up to whole circuitries can be modulated by light. Common optogenetic tools like Channelrhodopsin2 (ChR2) or Archaerhodopsin (Arch) are able to activate or silence neurons, or increase or inhibit transmitter release at axon terminals projecting to distinct brain regions ${ }^{1,2,3,4}$. However, Arch needs to be used carefully as it was shown that its activation at presynaptic terminals increases spontaneous transmitter release ${ }^{5}$. Arch is an outward rectifying proton pump that changes the $\mathrm{pH}$ value inside the cell. This alkaline milieu induces calcium influx and enhances transmitter release ${ }^{5}$. To specifically modulate intracellular signaling pathways, receptor chimeras composed of a light activatable optogenetic tool, such as rhodopsin or cone opsin, in conjunction with an adequate Gprotein coupled receptor, can be created $6,7,8$. The amount and variation of optogenetic tools available has increased significantly during the last decade ${ }^{9}$.

The purpose of optogenetics is to manipulate neuronal circuitries during behavior. Optogenetics enables, for example, the measurement of acute behavioral changes such as changes in anxiety behavior. Optogenetic tools are delivered into target regions of the brain via viral vectors. With the help of special promoters and enhancers, or the Cre-loxP system, cell type specificity can be ensured for the expression of optogenetic tools (Figure 1A). There are several genetically modified mouse lines expressing the enzyme Cre-Recombinase in specific cell types only. For example, Nex-Cre mice express the Cre-Recombinase in pyramidal neurons in the cortex and the hippocampus under the control of the Nex-promotor ${ }^{10}$. This enzyme is able to invert DNA-sequences, which are flanked by loxP sides $^{11}$. Consequently, the DNA-sequence of a double-floxed optogenetic tool, which is inverted and flanked by loxP sides, can only be transcribed by neurons that possess the CreRecombinase, but not by other neuronal types ${ }^{12,13}$. In the case of Nex-Cre mice, the optogenetic tool will be solely expressed in pyramidal neurons. Light stimulation of certain brain regions is then achieved via chronic implantation of optical fibers directly above the region of interest. Animals can then be coupled to a suitable light source and freely behave in nearly all kinds of behavioral tests. 

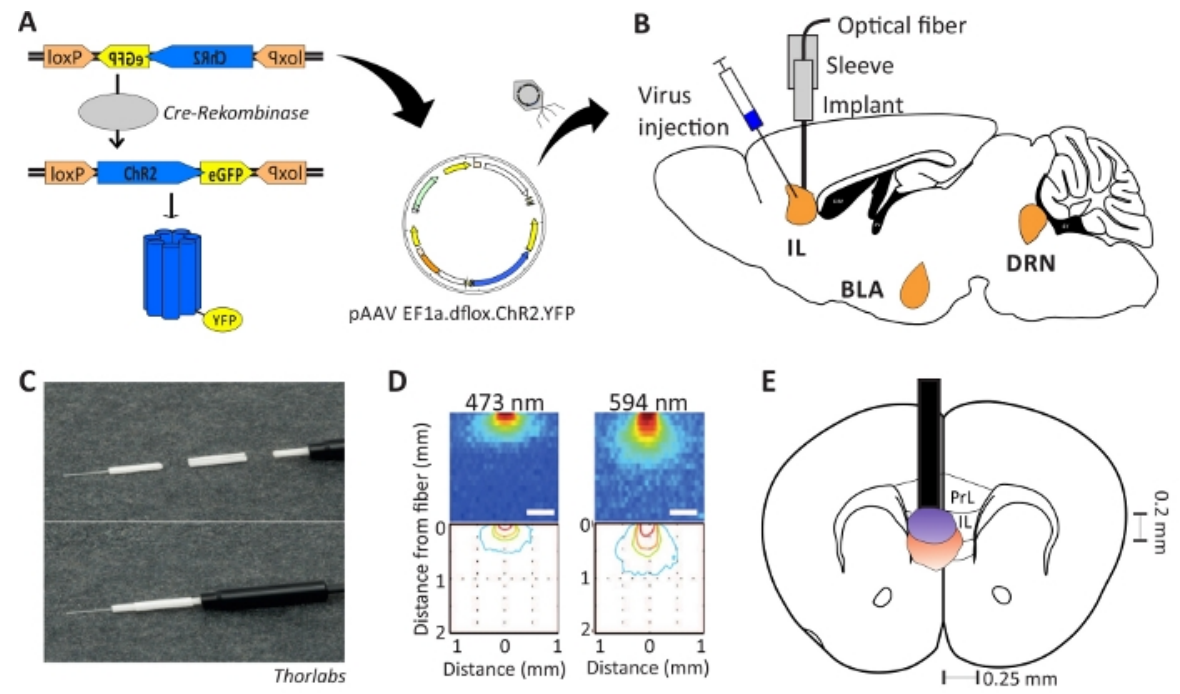

This figure has been modified from Berg et al. 2019 PLoS One

Figure 1: Injection and implantation. A) Cre-loxP system for ChR2-YFP. Double floxed optogenetic tool is packed in an adeno associated virus (AAV) for injection into the brain tissue. B) Sagittal view of the virus injection and implantation of an optical neuronal interface into/above the IL region of the mPFC. Injection and implantation were done from above. All regions of interest, IL, BLA and DRN, are shown. C) Detailed view of the implanted optical fiber, sleeve and light source. D) Spreading of blue and red laser light stimulation in grey matter brain tissue from a $200 \mu \mathrm{m}$ light fiber (Yizhar et al. 2011). Blue light spreads, at maximum, $0.5 \mathrm{~mm}$ into the tissue, red light about $1 \mathrm{~mm}$. Color coding: red $50 \%$, yellow $10 \%$, green $5 \%$, blue $1 \%$ if light reaches this area. E) Coronal view of the unilateral implantation directly above the left IL with a $200 \mu \mathrm{m}$ optical fiber. The IL region has a width of $0.25 \mathrm{~mm}$ in each hemisphere and a depth of $0.2 \mathrm{~mm}$. Blue and red light bulbs are the boarder of $5 \%$ light spreading and are transferred from Yizhar et al to the right size. LoxP: locus of X-over P1; ChR2: Channelrhodopsin; YFP: yellow fluorescent protein; dflox: double floxed; IL: infralimbic cortex; BLA: basolateral amygdala; DRN: dorsal raphe nuclei; PrL: prelimbic region. This figure has been modified from Berg $2019^{48}$. Please click here to view a larger version of this figure.

Optogenetic approaches are utilized as it enables both high temporal and spatial resolution ${ }^{14}$ and cell type specific modulation. Additionally, it is possible to repetitively use the implanted device without further treatment. After a stereotactic surgery, where the injection of an adenoassociated virus carrying the optogenetic tool and the implantation of the optical fiber is performed, mice can recover for two weeks. We have chosen a recovery time of only
2 weeks, because this is enough time to recover from the surgery and for the virus to express. As the behavioral experiments are followed by immunohistochemistry, we have to ensure that mice do not get too old during the experiment; otherwise the tissue quality is decreased. They show no obvious behavioral impairments from the implant and engage in typical cage behavior. Of course, the implantation is accompanied by a significant surgical lesion; therefore, the 
mice are monitored intensively. After the surgery, mice need to be single housed, as group housed mice tend to injure each other's fresh wounds and implants. However, housing conditions have a great impact on the anxiety level of male mice, as single housed mice show lower anxiety levels ${ }^{15}$ and in general less depressive-like symptoms ${ }^{16}$.

Chemical or electrical manipulation of brain circuitries lack the high cell type specificity of optogenetics and have a lower temporal and spatial resolution ${ }^{14,17,18}$. Depending on the experimental question, electrical or chemical stimulation can have different advantages. When passing fiber terminals in a specific region also need to be stimulated, electrical stimulation is the best method. Chemical stimulation is a good choice for when transmitter specific receptors in a whole region should be activated by agonists. Another great advantage of optogenetics compared to chemical or electrical stimulation is that endogenously, neurons are not sensitive to light, which avoids the occurrence of side effects ${ }^{19}$. Indeed, high light intensities might induce heating effects ${ }^{8,20}$, but due to proper control groups, the behavioral effects due to optogenetic manipulation can be eliminated.

Investigating rodent behavior, especially in regards to psychiatric diseases, has greatly improved with optogenetics in freely moving animals, as it enables the direct modulation of single receptors up to specific cell populations ${ }^{21}$ and circuitries $^{22}$. The possibility to measure the acute effects of such modulations, as well as the long-term behavioral effects after a defined time ${ }^{23}$ or after chronic stimulation ${ }^{24}$, enables a wide flexibility of experimental designs and provides very detailed insights into brain circuitries. Light stimulation can be used to modulate neurons located at the injection site of the optogenetic tool. When both the injection and implantation address the same brain region, cell bodies and back projecting axons of principle neurons and interneurons in this region can be targeted $3,6,8$. However, the light fiber can also be implanted in a region different from the injected one. In this case, light stimulation can modulate transmitter release at axon terminals in projection areas of the injected region $25,26,27$.

In the study here, optogenetics is used in combination with experiments to analyze anxiety-related behavior. Anxietyrelated psychiatric diseases affect more than one third of the world's population $28,29,30$ and cause a high economic burden $^{31}$. Those affected suffer from a feeling of arousal, tension and worry followed by avoidance behavior 32,33 . These chronically occurring negative emotions, which are mainly focused on future events ${ }^{34}$, strongly interfere with the daily life of patients. Common treatments like benzodiazepines or selective serotonin reuptake inhibitors (SSRIs) are only successful in some of the patients. A large amount of people do not respond to the treatment at all $^{35}$, showing that the mechanism underlying such diseases is not yet fully understood. The medial prefrontal cortex (mPFC) is known to play an important role in the development and manifestation of anxiety $21,25,27,36,37,38$. Specifically, the overactivation of the infralimbic cortex (IL) region in the mPFC might be part of anxiety-related disorders 39,40 . The example experiment described here could help to understand how modulations in the IL region of the MPFC influence anxiety behavior. Additionally, the development of new therapeutic strategies for anxiety-related psychiatric diseases can also potentially be supported.

2-6 month old male Nex-Cre mice are used to express ChR2 specifically in pyramidal neurons within the IL region of the mPFC ${ }^{41}$. Nex-Cre mice have a C57BI/6 background and express the enzyme Cre-recombinase specifically in 
pyramidal neurons. During a stereotactic surgery, double floxed ChR2-DNA is injected into the IL region via adeno associated viral vectors. The optical implant is placed directly above the region of interest (Figure 1B) and the implant is fixed with dental cement. Control animals receive an injection of double floxed tdTomato-DNA in the same region to mimic cell specific expression.

Animals are group-housed until the day of surgery and afterwards are single housed to avoid injuries from other mice. Mice are housed in individual ventilated cage (IVC) racks in Typl-L cages for single housed mice. The light-dark cycle follows a $12: 12 \mathrm{~h}$ rhythm, the light phase starting at 10 AM. All behavioral experiments are performed in the dark phase, which resembles the active phase of rodents. Water and standard food pellets are available ad libitum. After two weeks of recovery, which ensures a sufficient expression of ChR2 in pyramidal neurons, mice are used for behavioral experiments.

The Open Field (OF) is a $50 \mathrm{~cm}$ × $50 \mathrm{~cm}$ squared maze with sandblasted $40 \mathrm{~cm}$ high walls. The ground is divided in 16 squares where the inner 4 represent the center. The measured behavior is: 1) time spent in the center, 2) number of center entries, and 3) total distance moved. During this experiment, there are 4 trials totaling 20 minutes. In trials 1 and 3, no light stimulation occurs, and in trials 2 and 4 , a $20 \mathrm{~Hz}$ stimulation with $5 \mathrm{~ms}$ light pulse and $1 \mathrm{~mW}$ light intensity of $473 \mathrm{~nm}$ is performed (Figure 2A). In the later trials, habituation to the testing area was taken into account, but the use of sham-injected control animals indicate how habituation is expressed.

The Barnes Maze is an experiment for learning and memory. It is a circular platform that is $92 \mathrm{~cm}$ in diameter and contains 20 equidistant holes around the circumference. 19 of the holes are closed and under one hole an escape box is presented. For 4 consecutive days, mice have 4 training trials to learn the location of the escape box. On the $5^{\text {th }}$ day, the escape box is removed, and mice are tested on how much time they need to find the correct hole. The measured behavior is: 1) Time until the escape box/correct hole is found, 2) Number of target visits and errors, and 3) Distance moved until in the escape box. The light stimulation in different groups is done either during acquisition or consolidation, which take place on the training days $1-4$, or during retrieval on the testing day, which is day 5 (Figure 2D). 

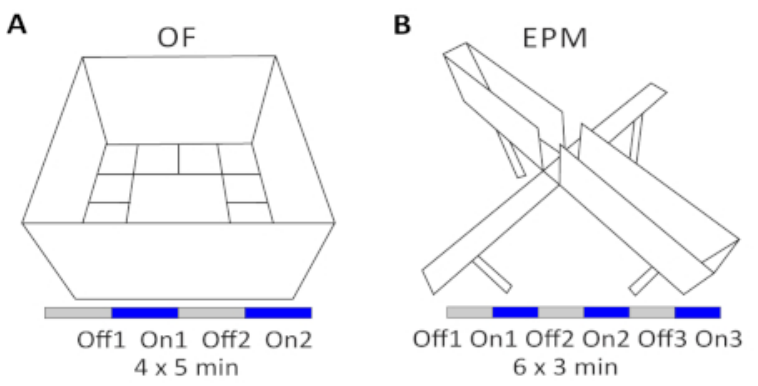

C

This figure has been modified from Berg et al. 2019 PLoS One

Figure 2: Behavioral experiments with optogenetic protocols. A) Schematic drawing of the Open Field experiment with the corresponding light stimulation protocol. C) Schematic drawing of the Elevated-Plus Maze experiment with the corresponding light stimulation protocol. D) Schematic drawing of the Barnes Maze experiment with the corresponding light stimulation protocol. EPM: Elevated-Plus Maze; OF: Open Field; BM: Barnes Maze Test. This figure has been modified from Berg $2019^{48}$. Please click here to view a larger version of this figure.

For optogenetic stimulation, the light intensity and frequency have to be adapted to the optogenetic tool and neuronal type that is under investigation. The lowest possible light intensity should be used in order to avoid damage to the tissue, as several studies have shown that there are possible heating effects due to strong light intensity 8,20 . For ChR2, a $20 \mathrm{~Hz}$ stimulation with a $5 \mathrm{~ms}$ light pulse is commonly used ${ }^{2}$. As ChR2 is quite light sensitive, $1 \mathrm{~mW}$ light intensity is sufficient. The light stimulation protocol alternates between light off and on trials to directly measure behavioral changes. The external room conditions for behavioral experiments should remain stable for the whole group of animals. Important conditions to consider are the noise (keep in mind that devices themselves might make noise), the smell (always clean the behavioral setups with ethanol), the light intensity, and the experimenter. The experimenter should always be the same person. Additionally, the time of day of the experiments should be the same for all animals in one group, a few hours after the start of the dark phase in the facility is preferred.
The goal of this experiment is to increase the excitation/ inhibition $(E / I)$ ratio in the IL region through strong activation of excitatory pyramidal neurons. An enhanced E/l ratio in this special cortex region is known to increase anxiety levels in mice $40,42,43,44$.

\section{Protocol}

Procedures involving animal subjects have been approved by the institutional animal research facility and the "Senatorin für Wissenschaft, Gesundheit und Verbraucherschutz" at the University of Bremen (\#146)

\section{Preparation of the optical implant ${ }^{9}$ ( Figure 1C)}

1. Place a ceramic ferrule flat side up in a bench vise.

2. Strip the coat of a $200 \mu \mathrm{m}$ diameter glass fiber with a fiber stripping tool and cut $2-3 \mathrm{~cm}$ long pieces with a ceramic fiber scribe. 
3. Place the piece of glass fiber into the ceramic ferrule with an even overhang on both sides.

4. Place a drop of superglue at the flat side of the ceramic ferrule with an injection canula.

NOTE: The protocol can be paused here.

5. Take the pre-implant out of the bench vise and on the round side of the ceramic ferrule, cut the glass fiber as short as possible with the ceramic fiber scribe.

6. Place the pre-implant at a ferrule polishing puck and polish the round side on 4 different polishing papers, by drawing an eight 20 times per paper $(30 \mu \mathrm{m}$ grit, $6 \mu \mathrm{m}$ grit, $1 \mu \mathrm{m}$ grit, and at last $0.02 \mu \mathrm{m}$ grit).
7. Take the pre-implant out of the ferrule polishing puck and cut the glass fiber on the flat side of the ceramic ferrule to the length needed for implantation. Begin measuring the length behind protruding superglue.

1. For an even cutting surface just scratch the glass fiber 2-3 times and then break it.

NOTE: Use the mouse brain atlas from Paxinos and Franklin ${ }^{45}$ to calculate the length of the implant. The implant must end directly above the region of interest and the thickness of the skull should be included in the length calculation. To stimulate the IL region, the glass fiber has a length of $1.8 \mathrm{~mm}$ (Figure 3 ).

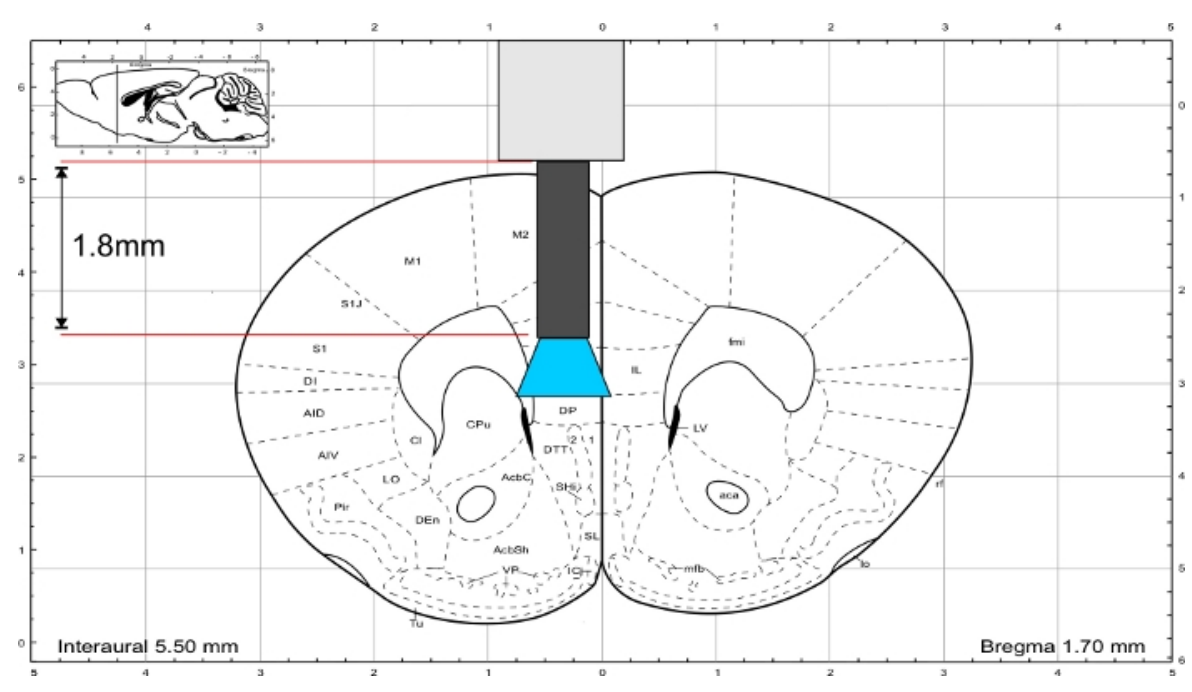

Figure 3: Mouse brain atlas (Paxinos and Franklin) with representative length of the implant to reach the IL region. Please click here to view a larger version of this figure.

8. Disinfect the finished implant for 10 minutes in ethanol and let it air dry before implantation.

\section{Injection and implantation}

1. Transport a single mouse to the surgical room and weigh it. Apply anesthesia with an intraperitoneal (i.p.) injection of Ketamine/Xylazine (Ketamine $0.12 \mathrm{mg} / \mathrm{g}$, Xylazine 0.01 $\mathrm{mg} / \mathrm{g})$.

1. Fix the mouse with the left hand and turn it on its back with the head held low. 
2. Target the left lower quadrant of the abdomen with the syringe and enter the injection canula $1 \mathrm{~cm}$ under the skin.

3. Inject anesthesia in a slow and constant motion into the abdominal cavity.

4. Place the mouse back into its cage and wait until it reaches a deep state of anesthesia.

NOTE: The depth of anesthesia can be determined by the absence of blinking and between-toes reflexes.

2. Place the mouse on a heating plate and fix the head in a stereotactic frame. Fix the nose and teeth in the front, and the ears on both sides.

NOTE: The head must be straight on the left-right and rostral-caudal axis to ensure correct stereotactic coordinates.

3. Apply analgesia with $2 \mathrm{mg} / \mathrm{kg}$ Carprofen subcutaneously into the back of the mouse and apply opaque eye ointment on both eyes to protect them from drying.

4. Moisten the hair on the scalp with a wet paper towel and then cut it off using scissors. Make sure to remove all the loose hair with the wet paper towel. To disinfect the scalp, use a cotton stick and take up $0.5 \mathrm{~mL}$ of a tincture containing iodine (Betaisodona $100 \mathrm{mg} / \mathrm{mL}$ Povidon iodine and $11 \mathrm{mg} / \mathrm{mL}$ iodine) and let it air dry.

NOTE: Instead of scissors, also an electric clipper can be used for proper hair removal.

5. Raise the scalp above the region of interest with a tweezer and cut $1 \mathrm{~cm}$ along the midline. Use two tweezers to push the skin aside to expose the skull. Make sure to also remove the thin skin above the skull and let the exposed skull dry.
1. Apply a $2 \mathrm{~mm} \times 2 \mathrm{~mm}$ drop of phosphoric acid (37\%) from the adhesive kit (e.g., Optibond) on the skull, distribute it with the tip of the syringe and let it take effect for $15 \mathrm{~s}$.

2. Remove all acid with a cotton stick and rinse the skull with $1 \mathrm{~mL}$ of $0.9 \% \mathrm{NaCl}$ twice.

3. Dry the skull with a cotton stick and compressed air. Caution: Phosphoric acid is dangerous and must be removed completely to avoid tissue damage.

7. Calculate the F-Factor for individual coordinates.

1. Place a glass canula in the stereotactic frame and locate it directly above bregma.

2. Zero the coordinate system and move the glass canula to lambda.

3. Calculate the F-factor ${ }^{46}$ with the following formula:

$\frac{\text { Bregma }- \text { Lambda }}{4.2}=F-$ Factor

4. Multiply the F-Factor with the coordinates from the mouse brain atlas to adjust them to the individual mouse.

8. Drill a hole in the skull for injection.

1. Use the adjusted coordinates to find the location on the skull directly above the structure of interest and mark it using the tip of an injection canula by scratching it above the bone surface.

2. Use the injection canula to drill a hole into the skull at the marked location by rotating the canula on the spot. If blood leaks out of the burr hole, rinse with 1 $\mathrm{mL}$ of $0.9 \% \mathrm{NaCl}$ and dry the skull afterwards.

9. Take up the virus solution into the glass canula.

6. Roughen the skull for later implantation. 
1. Place a drop of $100 \mu \mathrm{L}$ of $0.9 \% \mathrm{NaCl}$ onto the skull and a piece of parafilm $(1 \mathrm{~cm} \times 1 \mathrm{~cm})$ on top, sterile side up.

2. Place $1-2 \mu \mathrm{L}$ of virus solution onto the parafilm and lower the tip of the glass canula into it.

3. Connect the glass canula to a syringe, apply minimal negative pressure and wait until the virus solution is taken up by the cannula (within seconds).

NOTE: It is important to stop the application of negative pressure, before air is picked up into the canula. Therefore, there will always be a small remnant of the virus solution.

10. Inject the virus solution into the region of interest.

1. Place the virus filled glass canula above the burr hole.

2. Slowly lower the canula into the burr hole and zero the z-coordinate when the tip of the canula is at the level of the skull.

3. Lower the canula carefully to the lowest position of the injection site.

4. Focus the binocular at the meniscus of virus solution within the canula.

5. Apply a small amount of positive pressure with the syringe until the meniscus is lowered marginally.

6. Let the virus spread for $2-3$ min before moving the glass canula upwards to the next position.

7. Apply the virus solution every $200-300 \mu \mathrm{m}$ throughout the region of interest.

8. Remove the glass canula very slowly and discard it after the final injection.

11. Prepare the skull for implantation with the adhesion kit (e.g., Optibond $\left.{ }^{T M} F L\right)$.
1. Dry the skull with compressed air.

2. Apply $5 \mu \mathrm{L}$ of primer (e.g., Optibond, $1-30 \%$ (Ethanol, Silicic acid, Glycerinphosphatdimethacrylat, 2(2-(Methacryloyloxy)ethoxycarbonyl)benzoesäure, 2Hydroxyethylmethacrylat)) with the corresponding stick and let it dry for $15 \mathrm{~s}$.

3. Apply $5 \mu \mathrm{L}$ of bond (e.g., Optibond, $15-20 \% \quad 2-$ Hydroxyethylmethacrylat $+1-2 \%$ Alkalihexafluorosilikat( $\mathrm{Na})$ ) with the same stick and cure it for $20 \mathrm{~s}$ with UV light $(420-480 \mathrm{~nm})$.

NOTE: It is essential that the skull is dry and that the primer and bond are applied in a very thin layer.

Caution: Do not directly look into the UV light, as UV light may harm eyes.

12. Position the implant directly above the region of interest.

1. Fix the implant in the corresponding holder.

2. Dry the skull with compressed air.

3. Position the tip of the glass fiber directly above the burr hole and lower it carefully.

4. Stop lowering the implant when the remaining bulb of superglue touches the skull. Do not exert pressure on the skull!

NOTE: If the Injection and implantation are being done in different regions (e.g., dorsal raphe and hippocampus), drill all the necessary holes after applying phosphoric acid, but before the 2-component adhesion, then follow the instructions as previously described (step 2.8-2.14).

13. Fix the implant.

1. Check if the skull is still completely dry. 
2. Apply fluid dental cement (e.g., Gradia direct flo) around the implant and in the surrounding area and cure for $20 \mathrm{~s}$ with UV light $(420-480 \mathrm{~nm})$.

NOTE: The amount of dental cement depends on the free skull area. The whole skull should be covered by dental cement.

3. Apply two more layers of cement and completely fill the free and dried skull area. Cure every layer with UV light (420-480 nm).

14. Finish the surgery.

1. Apply $0.5 \mathrm{~g}$ iodine ointment (Betaisodona $100 \mathrm{mg} / \mathrm{mL}$ povidone iodine and $11 \mathrm{mg} / \mathrm{mL}$ iodine) at the whole wound

2. Inject $0.1 \mathrm{~mL}$ of glucose dissolved in $0.9 \% \mathrm{NaCl}$ subcutaneously into the neck for quick recovery.

3. Release the nose and ear fixation, bring the mouse into a fresh cage and place it under a heating lamp to avoid the loss of body heat.

4. When the mouse wakes up, bring it back into the facility.

5. Check its health status at least once a day. Take appropriate action if mice display any bad constitutions (e.g., ensure post-operative analgesia with Carprofen up to 3 days if mice display any signs of pain).

NOTE: After two weeks of recovery, mice can be used for behavioral experiments.

\section{Setting up a new experiment (Example ChR2 stimulation and Open Field)}

1. Pulser
1. Program the pulser (e.g., Prizmatix) for light stimulation.

2. Open the software and select the USB COM port that the light source is plugged into.

3. Choose Select Operation Mode (3) | Execute pulse sequence after trigger $\mathrm{HIGH}$, then stop when LOW to allow an external software to control the light source.

4. Program the light protocol. For a $20 \mathrm{~Hz}$ stimulation with $5 \mathrm{~ms}$ light pulse: choose $\mathrm{TI}=23 \mathrm{~ms}, \mathrm{P} 1 \mathrm{D}=5 \mathrm{~ms}$, $\mathrm{P} 1 \mathrm{I}=22 \mathrm{~ms}$ and $\mathrm{P} 2 \mathrm{D}=0 \mathrm{~ms}$.

5. Press Start Sequence. This status will remain until experiments are finished.

NOTE: The pulser software (Prizmatix Pulser) must be launched before the video tracking software; otherwise video tracking software will not be able to recognize the device.

2. Video tracking software (e.g., Ethovision XT)

1. Create a new experiment from a pre-defined template.

1. Open the software, go to File, choose New from Template. Select Apply a pre-defined template.

2. Choose Live tracking and select the camera by pressing on Source and confirm the connected Basler GenICam.

NOTE: The live image of the camera will now be displayed in the window on the upper right.

3. Press Next and choose the animal which should be recorded (Rodents, Mouse).

4. Press Next and select the arena template Open Field, square. Select the zone template Center, Border, Corners and confirm with Next. 
5. Confirm 1 subject that should be tracked with Next.

6. Select Center-point, nose-point and tail-base and confirm the animal color compared to the background as darker with Next.

7. Confirm the recommended sample rate of 12.5 with Next and finish the step.

8. Name the experiment appropriate and choose a location to save.

2. Define the experimental settings.

1. Go to Setup and Experimental Settings. Choose Center-point, nose-point and tail-base detection as Tracked features.

2. Select Use of Trial Control Hardware and go to Settings.

3. Select Noldus USB-IO box and confirm with Ok.

4. Choose Custom Hardware as Device Type at the TTL Port, which has been connected to the pulser device, and confirm with Ok.

3. Define the arena settings.

1. Go to Arena Settings and select Arena Settings 1.

NOTE: The camera will now automatically open a background image.

2. Confirm the image with Grab.

3. Adapt the pre-defined zones to the real arena by changing their size. Use the arrow and the two symbols on its right. If some zones are unnecessary, delete them.
4. Press 1. Draw Scale to Calibrate and pull a line from one corner of the maze to the other. Enter the length of the real distance in $\mathrm{cm}$.

5. Repeat that for the other axis.

4. Test if the light stimulation is working.

1. Go to Arena - Hardware Mapping and select Test on the grey bar.

2. Select Command Output 1 High and press Test. NOTE: There should be light emitting from the end of the optical fiber. When selecting Output 1 Low and Test, the stimulation should stop.

5. Define the trial control settings for 20 minutes of experiment. Set trials Off1, On1, Off2 and On2 to each be 5 minutes long.

1. Go to Trial Control Setting and select Track duration 30 minutes.

2. Prepare the main rule by adjusting the Condition: Time to 20 minutes by Selecting Settings and change 30 to 20 minutes. Confirm with Ok.

NOTE: The condition for start track should be when subject is in arena for $\mathbf{2}$ seconds. That way the system will automatically start tracking when the mouse is in the arena.

3. Create a sub-rule for the light stimulation: Go to Structures, more and select Sub-rule.

4. Give it a name such as light stimulation protocol.

5. Place it below the main rule and spread out the two boxes by selecting the blue area with the mouse courser. 
6. Go to Conditions, Time and give it a name like light on 1.

7. Adjust Condition is met with after $\mathbf{5}$ minutes. Confirm with Ok.

8. Place the box directly behind the Rule Begin box of the sub-rule by pulling it to the black line.

9. Go to Action | Custom Hardware and name it: light ON 1.

10. Select Action to perform as Output 1 High and confirm with $\mathbf{O k}$.

11. Place the box directly behind the Condition box. NOTE: Now after 5 minutes of the experiment the light stimulation should start.

12. Repeat the steps to define the time condition After 5 minutes and the action Output 1 Low to stop the light stimulation after another 5 minutes.

13. Repeat the steps again to program another light Off and light On trial.

14. Go to Structures | Sub-rule reference and check that the reference belongs to the correct sub-rule.

15. Choose Start conditions as Without delay and Stop conditions as Execute once per start condition. Confirm with Ok.

16. Place the reference box between action box 1 and condition box 2 of the main rule and draw a line from Action - start track to the Reference.

NOTE: Now the main rule directly starts the subrule after starting the track.

6. Define the detection settings to show the system what it should track.
1. Go to Detection settings and select Detection settings 1 .

2. Place a test mouse into the arena and select Automated Setup.

3. Choose Rodent as animal type and use the mouse curser to draw a box around the mouse in the arena. Confirm the Results OK? question with Yes.

7. Define the trial list for all experimental animals that should be tracked.

1. Go to Trial List and plan all animals to record today: Select add trials and select a number.

2. Select all the conditions defined before for every mouse.

3. Name the Animal-ID and the Treatment correctly to later simplify the analysis.

NOTE: The Animal-ID is irrelevant for the system and only important for later data analyzation by the experimenter. The grouping in Treatment and Control group is important for the system to know how to group and how to compare all the tracks in later analyzing steps.

8. Go to Acquisition and start with the experiment.

\section{Open Field experiment (anxiety)}

1. Bring the experimental mouse to the behavioral room right before the experiment to ensure a proper level of anxiety. NOTE: The behavioral experiments should be performed during the dark phase when mice are awake, and always in the same time slot to ensure comparability.

2. Couple the mouse via a sleeve to the light source by pressing it gently onto the grid of the cage. 
3. Place it into a waiting cage with fresh litter for $10 \mathrm{~min}$ to get acclimatized to the light cable.

4. Start acquisition by pressing the Start button in the video tracking software (e.g., Ethovision XT).

5. Transfer the mouse from the waiting cage into the left upper corner of the open field. Remove the arm within 2 seconds to avoid tracking an arm instead of the mouse.

6. Leave the visual field of the mouse during the experiment and keep calm.

7. After 20 minutes, when the experiment is finished, remove the mouse from the maze, disconnect the light cable and put it back into its home cage.

8. Bring the mouse back into the facility.

\section{Barnes Maze (learning)}

1. Bring all experimental mice into the behavioral room around 1 hour before the experiment.

2. Prepare the Barnes Maze by closing all holes except one, under which an escape box is placed. Place a wall of carton in the middle of the platform, which is the starting area for the mouse.

3. Connect one mouse to the light source (sleeve on a light cable) at both implants.

4. Place the mouse directly into the middle of the Barnes Maze into the wall of carton, which prevents the mouse from running around before the experiment starts.

5. Press Start in the video tracking software (e.g., Ethovision $\mathrm{XT}$ ) and remove the carton.

NOTE: The software tracks the mouse until the correct hole is reached but be prepared to stop the trial manually just in case the software does not recognize the hole transition.
6. Take the mouse out of the maze and remove the connection to the light cable.

NOTE: If this is a training day with several trials per mouse, leave the mouse in the waiting room next to the behavioral room until the next training session starts. If this was the testing day with only one testing trial per mouse, bring the mouse back to the facility.

\section{Data analysis (Example Open Field data with 4 distinguishable trials)}

1. Video tracking software (e.g., Ethovision XT)

1. Define the experimental groups and trials in the data profile.

1. Go to Data Profiles on the left and choose Treated vs. Control.

2. Go to Nesting in the new window on the middleleft and select Trial control state.

3. Choose the state interval from the Element Action: start Track to the Element Action: light goes ON 1.

4. Place the Nesting box between the Filter box Treatment and the corresponding result box.

NOTE: This defined interval is Off1, which describes the first 2.5 minutes of the experiment where no light stimulation is present.

5. Repeat the steps for intervals On1 (from element Action: light goes on 1 to the element Action: light goes out 1), Off2 (from the element Action: light goes out 1 to the element light goes on 2) and On2 (from the element Action: light goes on 2 to the element Action: stop track).

6. Repeat the 4 intervals for the control filter group. 
NOTE: Every nesting box needs its own result box with the names Off1, On1, Off2, On2. Now both the treatment and control group are divided into 4 different light stimulation trials which are analyzed separately.

2. Define the parameters to analyze in the analysis profile.

1. Go to Analysis Profiles on the left and select In zones.

2. Select the Dependent Variable In Zone and select Center as zone.

3. Double click on In center and choose any of selected points and select only in center.

4. Before leaving the window go to Trial Statistics and select Frequency, Cumulative duration and Latency to first.

5. Add the Dependent Variable Distance moved. NOTE: In Group Statistics, choose whether to use the standard error or standard deviation as error. With this profile, the data for Time spent in the center, Center entries and Total distance moved is available.

\section{Extract data}

1. Go to Results and select Statistics and Charts.

2. Press Calculate to see the analyzed data.

NOTE: The trial statistics gives information about every single mouse and group statistics analyzes the mean and error for both groups, divided into 4 trials with the corresponding bar plot.

3. Press Export data and select the trial statistics and the location to save.
NOTE: The exported data is saved as an Excel file and with individual values for every mouse. In this Excel file the Animal-ID helps to identify the mice.

4. Go to Heatmap Visualization and press Plot Heatmaps.

5. Select Trials on the right to see individual heatmaps for every mouse and trial.

6. Do a right click on the mouse and export heatmaps as images.

2. Plotting

1. Open the spreadsheet file at the computer and calculate the means and standard errors (SEM) for all 4 trials in every measured condition and group.

2. Generate graphs in a statistics program (e.g., Sigma Plot).

1. Copy the means and SEM into the correct order from the spreadsheet file to Sigma Plot. The rows have to contain the data for Off1, On1 etc. and the columns contain trial, mean and SEM as heads.

2. Select all three columns and go to Create Graph.

3. Select the Bar box and choose ungrouped bars with error (upper row, third box).

4. Confirm with Finish to open a new graph page.

5. Label the whole graph, then go to Home, select the Graph box on the left and press Export. Select a destination folder and choose MetaFile (*.wmf) as format.

NOTE: The .wmf format can be processed later in a graphical software like CoreIDraw.

3. Calculate statistics for obtained data. 
1. Copy raw data from the spreadsheet (Off1, On1 etc.) into single columns of Sigma Plot.

2. Mark the columns to compare and go to Analysis, choose t-test and press Run.

3. Confirm the data format Raw with Next and run the test with Finish.

\section{Representative Results}

The aim of this protocol is to measure changes in the behavior of genetically modified mice during an optogenetic experiment. Optogenetic manipulation is done by injection of an adeno associated viral vector. Light stimulation in freely moving mice is possible via implantation of a light fiber directly above the region of interest.

In Figure 4, the results of an optogenetic experiment are presented. A strong activation of excitatory pyramidal neurons in the IL region via ChR2 increased anxiety-related behavior in the Open Field. ChR2 was injected in the IL region of the mPFC in Nex-Cre mice for expression in pyramidal neurons (Figure 4A). During two anxiety tests, the Open Field (Figure 4B,C) and the Novelty-Suppressed Feeding test (Figure 4F,G), ChR2 is stimulated with blue light and activates pyramidal neurons. As control, another group of mice received an injection of the fluorophore tdTomato instead of ChR2 (Figure 4D,G). In such experiment, anxiety is defined as avoidance of the brighter central area. Mice show an intrinsic avoidance of open areas because they are anxious of predators.

In the Open Field experiment, shown in Figure 4B, mice executed 4 trials of 5 minutes each. In trials 1 and 3 no light stimulation occurred $(\mathrm{Off} 1,2)$ and in trials 2 and 4 , blue light stimulation with $20 \mathrm{~Hz}$ (5 ms light pulse) and $1 \mathrm{~mW}$ intensity was performed $(\mathrm{On} 1,2)$. The heatmaps show that, in the experimental group, the center duration differed between Off and On trials. During light stimulation, mice preferentially stay in the border zone. Control animals also prefer the border, but do not change their behavior upon light stimulation. In Figure 4C, the main behavioral measurements during the Open Field experiment are shown for the experimental group. If the data passed the Shapiro-Wilk test for normality, statistics were done with an independent two-tailed t-test. If the normality test failed, the Mann-Whitney-Rank Sum test was used as non-parametric alternative. For these kinds of experiments, a within group comparison was chosen to investigate if light stimulation can directly change anxiety behavior over time, independent of the baseline anxiety of experimental and control animals. The center duration decreased significantly during both light stimulation trials, indicating increased anxiety levels. The total distance moved was not altered, showing that locomotor behavior was not affected. The number of center entries was increased, although not significantly. In Figure 4D, the data of the control group is shown. Control animals did not display any behavioral changes between Off and On trials in any of the analyzed parameters, showing that light stimulation or implantation did not cause the observed effects. In sum, this test shows increased anxiety during light stimulation of IL pyramidal neurons via ChR2.

In Figure 5, the data of an unsuccessful optogenetic experiment is shown for the Elevated-Plus Maze. During the Elevated-Plus Maze experiment, which is presented in Figure 5A, mice completed 6 trials of 3 minutes each. In trials 1, 3 and 5 no light stimulation was performed (Off1, Off2, Off3) and in trials 2, 4 and 6, blue light stimulation with $20 \mathrm{~Hz}$ (5 ms light pulse) and $1 \mathrm{~mW}$ intensity was performed (On1, On2, On3). In these exemplary results, the length of the optogenetic protocol and the construction of the maze itself 
were not suitable for the transgenic mouse line. In Figure $\mathbf{5 B}$, it can be seen, that several mice slipped off the maze with their back paws or even fell down. When this happened, mice got a second chance to perform the EPM one day later. If they fell down again, they were excluded from analysis. When mice slipped off several times but managed to stay on the maze, data was analyzed normally. Nevertheless, the data has to be interpreted very carefully and control animals get greater importance. Nex-Cre mice had motor difficulties to stay on the narrow open arms. To avoid this, little walls, with a height of $1 \mathrm{~cm}$, would have helped for a secure hold of the back paws on the arms of the maze. Both the heatmaps and the graphs show that experimental, as well as control mice, started to avoid the open arms from trial 2 (On1) on (Figure 5C-E). The time on the open arms is significantly decreased for both groups, as are the open arm entries. Analyzing the experimental group only obtained data implicating a large anxiogenic effect of the light stimulation, as time on the open arm and open arm entries are significantly decreased during the On1 trial. However, when comparing this data to the control group, which show the same behavior, it becomes clear that the observed behavior is not mediated by the optogenetic stimulation, but by avoidance of the open arms in general due to habituation to the maze. This data underlines the importance of a proper control group to distinguish between behavioral effects mediated by optogenetic stimulation and possible behavioral adaption. Also, this data sheds light on the importance of properly adapting an experimental setup to suit the specific mousse line and experimental question.

To validate and strengthen gathered behavioral data, brains of mice are removed after the last experiment to control for the correct injection and implantation (Figure 6). Brains are fixed in $4 \%$ paraformaldehyde and removed from the skull. Brains are dehydrated in 30\% sucrose for 1-2 days and cryosliced afterwards. The $40 \mu \mathrm{m}$ thick coronal brain slices are washed and mounted on superfrost objective slides with a mounting medium containing DAPI, which stains cell nuclei. This enables the identification of target areas in the coronal slices. Fluorescence of the YFP-tag or tdTomato itself indicates the location of the virus injection. In Figure 6B the exemplary injection sites of ChR2-YFP on the left (yellow), and tdTomato on the right (red) are presented. With the help of a template, adapted from the Paxinos and Franklin 45 mouse brain atlas, the IL region can be identified. In both slides, the optogenetic tool is expressed in the IL region, but also in adjacent brain regions. For a proper interpretation, the spreading of blue light in brain tissue is consulted ${ }^{8}$ (Figure 1D,E). It can be seen that the blue light will reach the DP region below the $\mathrm{IL}$ with only less than $5 \%$ of the initial 1 $\mathrm{mW}$ light intensity at the fiber tip (Blue line in Figure 1D) ${ }^{8}$. Additionally, small amount of light can go upward to the PrL region due to back-scattering 47 . Consequently one can say that the IL region is illuminated the most strongly, however adjacent regions like the $\mathrm{DP}$ and $\mathrm{PrL}$ region may also be slightly stimulated. Therefore, IL-cell specific stimulation is not guaranteed and immunohistochemical analysis of the adjacent regions should be performed, to see if the activity of PrL and DP cells is modulated via light. In Figure 6C, another important control is shown: the specificity of the NexCre mouse line. Via antibody staining against the two cell types in the IL region, glutamatergic principle neurons and GABAergic interneurons, it can be seen, that the ChR2-YFP expression only occurs in glutamatergic neurons and not with GABAergic ones.

All in all, our experiments show that with optogenetic manipulation during behavioral testing, changes in anxietyrelated behavior could be observed. By using more than 
one test for the same behavior, a reliable conclusion can be drawn. In addition, immunohistochemical analysis confirms the obtained data. Our experiments suggest, that the specific activation of pyramidal neurons in the infralimbic cortex increased anxiety-related behavior in certain assays.
A
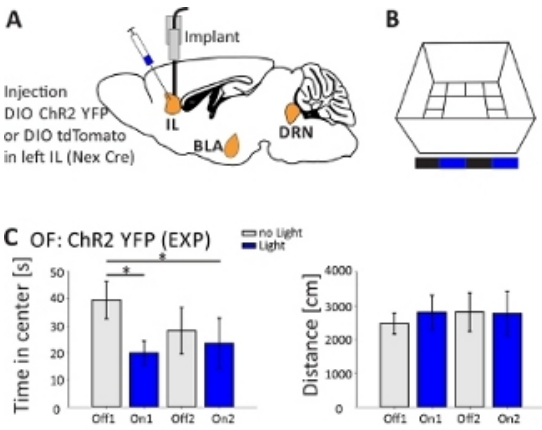

Enovent

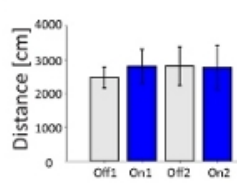

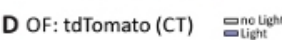

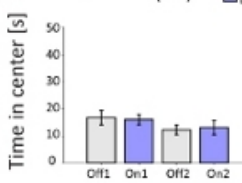

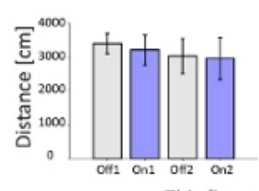

This figure has been modified from Berg et al. 2019 PLoS One

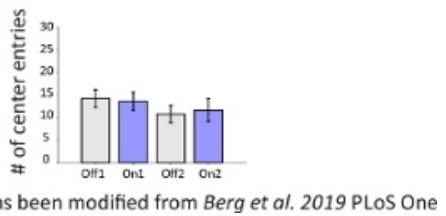

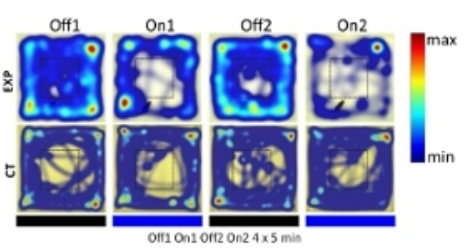

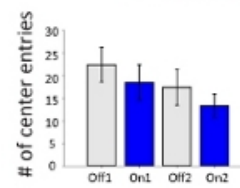

Figure 4: Optogenetic activation of IL pyramidal neurons increases anxiety behavior. Light stimulation during experiments: $473 \mathrm{~nm}, 1 \mathrm{~mW}, 20 \mathrm{~Hz}$ stimulation. A) Schematic drawing of injection and implantation site for ChR2-YFP or tdTomato into the IL. During the experiment, pyramidal neurons in the IL region of the MPFC are activated by ChR2. Saggital brain slices adapted from the Paxinos and Franklin mouse brain atlas, saggital: lateral o,6. B) Open Field maze with light stimulation protocol (20 min with 4x5 min alternating Off and On trials; left) and heat maps of exemplary ChR2-injected(EXP) and tdTomato-injected (CT) mice in all 4 trials of the experiment (right). EXP animals spend less time in the center of the OF when stimulated with blue laser light. For CT animals, time spent in the center does not differ between light Off and On trials. C) Group data for EXP animals in the OF, $n=11$. Mice spend significantly less time in the center of the OF when

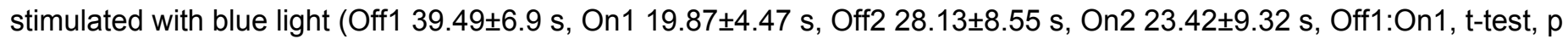
$=0.033,{ }^{*} ;$ Off1:On2, MWRS, $p=0,049,{ }^{*}$ ). Distance moved is not affected (Off1 2703.09 $\pm 292.65 \mathrm{~cm}$, On1 3113.4 \pm 491.15 $\mathrm{cm}$, Off2 $3331.86 \pm 482.62 \mathrm{~cm}$, On2 $3082.17 \pm 658,61 \mathrm{~cm})$. \# of center entries decrease with time, but show no significant differences (Off 1 22.36 \pm 3.78 , On1 18.45 \pm 3.95 , Off2 17.36 \pm 1.99 , On2 13.27 \pm 2.64 ). D) Group data for CT animals in the $\mathrm{OF}, \mathrm{n}=15$. Time mice spend in the center of the OF, the distance moved, \# of center entries does not change between light On and Off trials (Time in center Off116.73 \pm 2.65 s, On1 $16.02 \pm 1.89 \mathrm{~s}$, Off2 $12.02 \pm 1.76 \mathrm{~s}$, On2 13.04 $\pm 2.58 \mathrm{~s}$; Distance Off1 $3399.69 \pm 296.77 \mathrm{~cm}$, On1 $3210.6 \pm 446.9 \mathrm{~cm}$, Off2 $3030.28 \pm 513.83 \mathrm{~cm}$, On2 2955 $\pm 617.7 \mathrm{~cm}$; \# of center entries Off1 14.2 \pm 1.98 , On1 13.6 \pm 2.02 , Off2 10.8 \pm 1.88 , On2 11.67 \pm 2.5 ). CT mice show significantly higher baseline anxiety (Off1 EXP:CT, MWRS, $\left.p=0.005,{ }^{* *}\right)$. Values are mean \pm S.E.M. * indicate significant differences $(p \leq 0.05),{ }^{* *}$ indicate significant differences ( $p \leq 0.01)$. t-test always two-tailed, MWRS: Mann-Whitney Rank Sum test; IL: infralimbic cortex; BLA: basolateral 
amygdala; DRN: dorsal raphe nuclei; OF: Open Field; CT: control animals; EXP: experimental animal; L: light. This figure has been modified from Berg et al. 2019, PLoS One ${ }^{43}$ and from Berg $2019^{48}$. Please click here to view a larger version of this figure. 

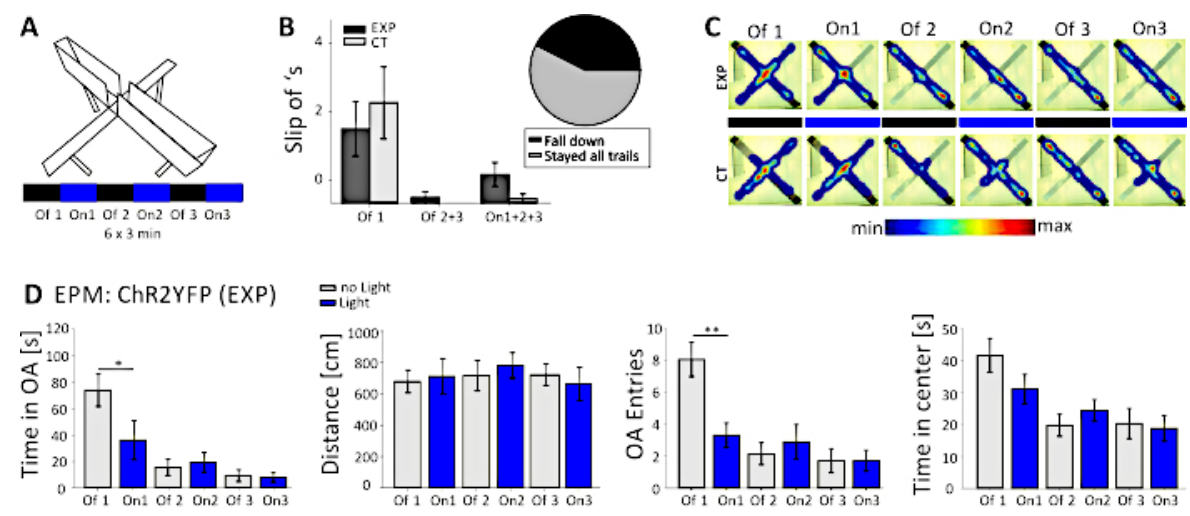

E EPM: tdTomato (CT) 品
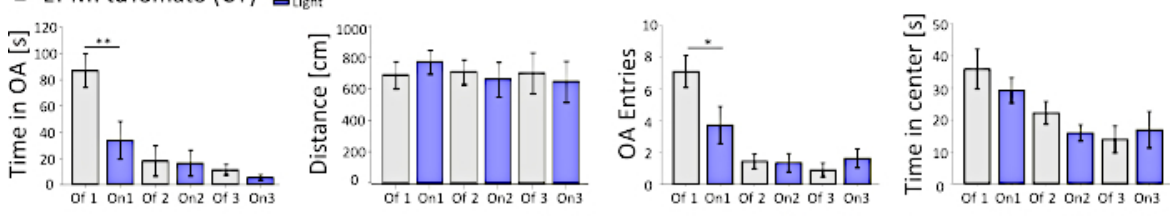

This $\mathrm{f}$ gure has been modif ed from Berg et al. 2019 PLoS One

Figure 5: EPM experiment failed to show behavioral effects in Nex-Cre mice. Light stimulation during experiments: $473 \mathrm{~nm}, 1 \mathrm{~mW}, 20 \mathrm{~Hz}$ stimulation. A) Elevated-Plus Maze with light stimulation protocol (18 min, 6x3 min, alternating Off and On trials). B) Group data for the mice that "slip off" is included into the data, total $n=23$. Nex-Cre mice had a tendency to slip off of the open arm with their back paws, independent of the experimental group (left). Only mice that stayed on the maze for all 6 trials were considered in later analyses. Slip off's in the first Off1 phase are reason for later avoidance of the open arms (Off1 EXP 1.63 \pm 0.6 , CT 2.2 \pm 0.79 , Off2+3 EXP 0.125 \pm 0.125, CT 0 \pm 0, On1+2+3 EXP 0.625 \pm 0.26, CT $0.1 \pm 0.1$ ). Pie chart (right) shows mice falling from the maze during the 18 min with $42.42 \%$. Only $57.57 \%$ finished the experiment. C) Heat maps of exemplary EXP and CT mice in all 6 trials of the experiment. Both groups show a decrease in open arm duration after the Off1 trial. D) Group data for EXP animals in the EPM, $n=12$. Time spent in the open arms decreased

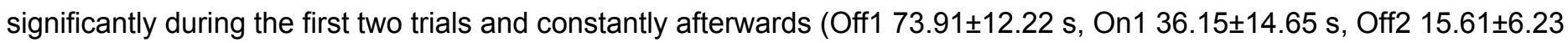
s, On2 $19.49 \pm 7.51 \mathrm{~s}$, Off3 $9.36 \pm 4.44 \mathrm{~s}$, On3 $7.96 \pm 3.47 \mathrm{~s}$. Off1:On1, t-test, p=0,041, *). The distance moved is not affected (Off1 $679.96 \pm 71.63 \mathrm{~cm}$, On1 $712.24 \pm 112.82 \mathrm{~cm}$, Off2 $717.49 \pm 97.39 \mathrm{~cm}$, On2 $782.51 \pm 81.11 \mathrm{~cm}$, Off3 $722.11 \pm 68.60 \mathrm{~cm}$, On3 $663.90 \pm 106.57 \mathrm{~cm}$ ). The amount of open arm entries decreases significantly from Off1 to On1 and then stays constant (Off1 8.08 \pm 1.08 , On1 3.33 \pm 0.76 , Off2 2.16 \pm 0.69 , On2 2.91 \pm 1.09 , Off3 1.73 \pm 0.75 , On3 1.73 \pm 0.66 . Off1:On1, t-test, $\left.p=0.002,{ }^{* *}\right)$. The time spent in the center of the EPM decreases along trials but shows no significant difference from Off to On trial (Off1 $41.71 \pm 5.34$ s, On1 $31.2 \pm 4.59$ s, Off2 $19.8 \pm 3.44$ s, On2 $24.49 \pm 3.38$ s, Off3 $20.37 \pm 4.77$ s, On3 $18.85 \pm 4.07$ s). E) Group data for CT animals in the EPM, $n=11$. CT data shows the same significant decreases as EXP data, indicating the experiment

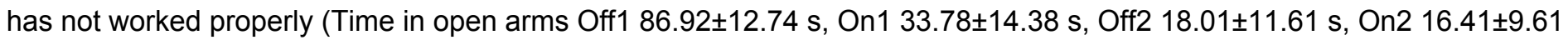
s, Off3 $11.36 \pm 4.01 \mathrm{~s}$, On3 5.43 \pm 2.07 s. Off1:On1, MWRS, p=0.009, **; Distance Off1 705.11 $\pm 88.36 \mathrm{~cm}$, On1 789.45 \pm 77.53 cm, Off2 $724.74 \pm 80.49 \mathrm{~cm}$, On2 $676.57 \pm 111.99 \mathrm{~cm}$, Off3 $716.99 \pm 132.47 \mathrm{~cm}$, On3 $663.03 \pm 132.46 \mathrm{~cm}$; Open arm entries 
Off1 7.09 \pm 1 , On1 3.72 \pm 1.17 , Off2 1.45 \pm 0.47 , On2 1.36 \pm 0.58 , Off3 0.91 \pm 0.43 , Off3 1.64 \pm 0.59 . Off1:On1, MWRS, $p=0.01$,

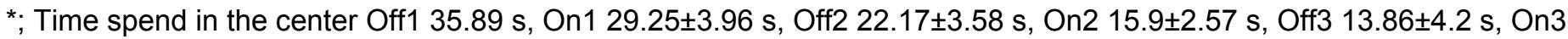
$16.89 \pm 5.75$ s). Values are mean \pm S.E.M. ${ }^{*}$ indicate significant differences $(p \leq 0.05),{ }^{* *}$ indicate significant differences $(p \leq 0.01)$. t-test is always two-tailed, MWRS: Mann-Whitney Rank Sum test; EPM: Elevated-Plus Maze; CT: control animals; EXP: experimental animal; OA: open arms. This figure has been modified from Berg et al. 2019, PLoS One ${ }^{43}$ and from Berg $2019^{48}$. Please click here to view a larger version of this figure.
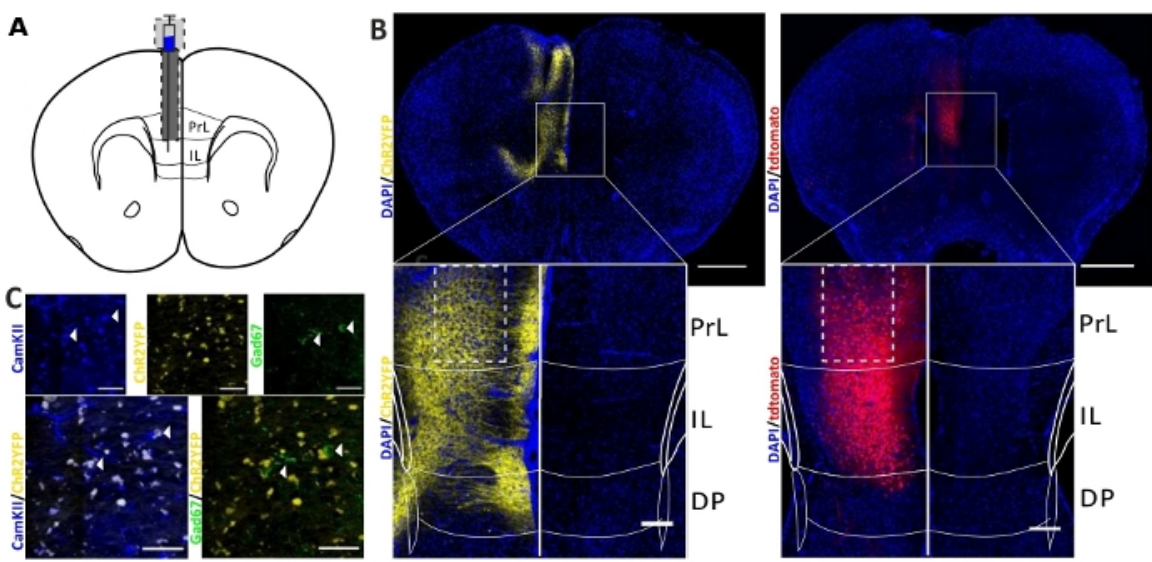

This figure has been modified from Berg et al. 2019 PLoS One

Figure 6: Injection side of ChR2 and tdTomato in the IL and Nex-Cre specificity. A) Schematic drawing of the implantation site on coronal brain slices at AP $+1.66 \mathrm{~mm}, \mathrm{~mL} 0.3 \mathrm{~mm}$, DV $-1.8 \mathrm{~mm}$, with unilateral injection and implantation (adapted from mouse brain atlas, Paxinos and Franklin, Bregma $+1.54 \mathrm{~mm}$ ). B) Exemplary injection sites of ChR2-YFP (left, yellow) and tdTomato (right, red) merged with DAPI stained cell nuclei (blue) in Nex-Cre mice. Scale bar $1 \mathrm{~mm}$. Insets show high magnification of IL region. Scale bar $150 \mu \mathrm{m}$. White boxes indicate location of insets. C) Top row: confocal images of the left IL region of a Nex-Cre mouse stained with CamKII as a marker for glutamatergic neurons (blue), and ChR2-YFP (yellow) or Gad67 as a marker for GABAergic neurons (green), of a Nex-Cre mouse. Bottom row: colocalization of ChR2YFP (yellow) with CamKII (left, blue), but not with Gad67 (right, green), showing specificity of Nex-Cre mice for glutamatergic neurons. Scale bar $50 \mu \mathrm{m}$. PrL: prelimbic cortex; IL: infralimbic cortex; DP: dorsal peduncular cortex. This figure has been modified from Berg et al. 2019, PLoS One ${ }^{43}$ and from Berg 201948. Please click here to view a larger version of this figure.

\section{Discussion}

Using light to manipulate neuronal signaling has been the method of choice for nearly one decade now. Since 2005 , the number of published articles about the development of new optogenetic tools $4,6,8,14,49,50,51$ and studies where such tools are utilized to investigate brain circuits $21,23,40,43,52$, highly increased. On one hand, with the enormous diversity of injectable optogenetic tools, implantation variants, transgenic mouse lines and behavioral experiments, the possibility for experiments is manifold and unlimited. On the other hand, the possibility to make faults in choosing experimental conditions 
is very high and the experiments are so specific, that often the comparability to other studies is difficult.

\section{Critical steps}

One important critical step of this protocol is proper planning. The choice of the optogenetic tool should match the scientific question. Is it only necessary to manipulate the overall activity of a neuron or synapse? Then commercially provided tools like ChR2 $21,25,27$ and $\mathrm{Arch}^{37}$ are a good choice. But apart from that, if one special neurotransmitter system or even a single receptor should be manipulated, an individual receptor chimera is often the better choice ${ }^{3,6}$. Several receptor chimeras with GPCRs, the so called Opto-XRs, and guidelines to produce them are already available ${ }^{4,50}$. Other than the choice of optogenetic tools, the mouse line in combination with the behavioral experiment is also critical. Different background strains, like for example C57BI/6 and BALB/cByJ, display different behavioral phenotypes in some respects ${ }^{53,54}$. C57BI/6 mice have a low baseline anxiety and can be used for anxiogenic manipulation, whereas BALB/cByJ show higher anxiety levels and are therefore more sensitive to anxiolytic drugs. Additionally, the transgenic variants of these background strains may also vary in their phenotype ${ }^{48}$. With a proper combination of specific promoters in conjunction with an optogenetic tool and transgenic mouse line, nearly every desired cell population can be targeted.

A critical step during surgery is targeting the correct location. With the help of the mouse brain atlas, proper coordinates for the anterior-posterior axis, and medial-lateral axis, and depth of the structure can be established ${ }^{45}$. In reality, every skull has a slightly different form and size. Thus, the F-factor ${ }^{46}$ to adjust the stereotactic coordinates is quite important, as is the correct nose and ear fixation during stereotactic surgery. If the head of the mouse is tilted, the injection canula will fail to target the desired region of interest.

Additionally, the diameter of the injection canula is also critical. If it is too small, no virus can be released into the tissue, if it is too wide, the canula will leak virus solution on its way to the region of interest. If the implanted optical fiber terminates directly above the target region, the virus expression in the cortex regions above does not matter. But if the implant is placed above other regions to stimulate axon terminals, the axons of upper cortex regions will also be activated by light and falsify obtained data. As an example: The IL region and the prelimbic (PrL) region both project to the basal amygdala ${ }^{55,56}$ but have completely different functions and roles in the modulation of anxiety ${ }^{26,57}$. If the implant is placed above the amygdala to activate axon terminals from the IL region, and during the injection virus solution was also placed into the $\operatorname{PrL}$ due to the wrong injection canula, the risk of also activating axon terminals from the PrL is very high.

During the preparation of the skull for the fixation of the implant, the sparse usage of primer and bond is crucial for a reliable and durable fixation. If the 2-component adhesion system is not applied thinly, the dental cement might detach from the skull after a couple of days or weeks. In addition, the skull also has to be completely dried before fixing the implant, as otherwise the cement will not attach properly to the skull.

Critical steps also exist in the behavioral part of this protocol. First, the construction of the maze is very important. In every behavioral setup, several variants exist in the literature regarding size and form, as well as for the procedure itself $58,59,60$. It is important to choose a variant that makes the data comparable and reproducible. Also, special requirements for utilized mouse lines should be taken into account ${ }^{43,48}$. In the representative data for the EPM it can be 
seen that several Nex-Cre mice fell from the maze or slipped off several times (Figure $\mathbf{2 b}$ ). For these mice, a maze with a small wall around the open arms would have been a better alternative.

Second, it is critical to keep all external room conditions constant $^{61}$, otherwise different groups of mice would not be comparable at all. In this regard, it is very important to choose the time of the experiment as one where the experimental setup is vacant and the experimenter is always present. Furthermore, events in the building, such as construction work, testing of any systems (fire alarm) or the cleaning day of the mouse facility, should be considered in order to avoid interference with the obtained data.

Finally, handling and housing conditions are critical for behavioral experiments. When an implantation is performed, mice need to be single housed because of the risk of injury from other mice. To ensure good comparability between groups and a low error within one group, every mouse needs to have the same cage size and enrichment. For anxietyrelated experiments, single housing has some advantages as singe housed male mice show a lower baseline anxiety level, less variation in their anxiety level, and less depressivelike symptoms ${ }^{15,16}$. Group housed male mice might strongly differ in their anxiety level because of hierarchy among the mice. Besides the housing, a constant and equal handling of all mice and groups is also important. Grabbing the mouse in order to connect the light fiber on the implant is very stressful. Therefore, this procedure has to be the same for every mouse, meaning the same technique and the same experimenter. Furthermore, the habituation time in the waiting cage, which is meant to calm the mouse down from the stressful connecting procedure, also needs to have equal conditions in duration, litter and position to the maze. The handling within the mouse facility is also critical for later behavioral performance. Experimental and control animals should not be cleaned on different days or by different people, as this is also stressful for mice. Additionally, the cleaning day should not be the experimental day to avoid differences in behavior.

\section{Troubleshooting}

There are several problems which might occur during the protocol. For example, drilling a whole in the skull during the stereotactic surgery could damage blood vessels. Usually, strong bleeding occurs, especially above bregma and lambda. If this happens, do not try to stop the bleeding with cotton sticks as they tend to extend even more bleeding out of the vessel because of their absorbency, instead, directly rinse with $\mathrm{NaCl}$.

It can also happen that the pressure injection of the virus solution is not working. In this case, it could be that parafilm, a scab from the burr hole or brain tissue, is clogging the tip of the canula. In this case, remove the canula slowly out of the brain without changing the $\mathrm{x}$ - or $\mathrm{y}$-axis and use a tweezer to remove 1-2 $\mathrm{mm}$ of the front part of the canula tip. Before lowering the canula again, test for functionality by applying small amount of pressure to see if virus comes out of the canula tip. To avoid constipation, lower the canula with a constant speed and do not stop the movement until the deepest depth of the injection side is reached. If too much of the canula tip is removed and the diameter is too large, the canula will damage tissue and the risk of applying the virus all at once will be increased. Thus, make sure that only the clogged part of the tip is carefully removed.

During the behavioral experiment, the setup of the experiment in the video tracking software (e.g., Ethovision XT) might cause problems. If, for example, the light output is not working 
properly, this can be due to several reasons. The Pulser has to be opened, programmed and started before Ethovision XT is opened. The hardware needs to be selected correctly in the "Experimental setup" (step 3.2.2.4). If the wrong IOBox, or anything other than "Costume Hardware" is selected, the Pulser device cannot be controlled by Ethovision. If the test of the light output is successful, but the programmed light protocol in "Trial control settings" does not work during acquisition, the sub-rule or sub-rule reference might be located incorrectly or the conditions and actions are unclear. For example: does the reference belong to the correct subrule? Is the reference programmed correctly (e.g., how often is the sub-rule executed)?

Additionally, it might happen that during "detection settings" the animal is adequately tracked, but during acquisition there are samples where the subject is not found. In this case, check if the illumination in the experimental room was changed, or if anything produced unwanted shadows within the maze. The entire bottom of the maze has to have the same color, as the setting will only work for one specific combination. If for whatever reasons different bottom colors or shadows can not be avoided, define the detection setting in the darkest part of the maze.

To change any settings after the acquisition of the first animals, do not apply these changes in the already used settings. Duplicate them to adjust them. This also means that the already recorded trial is not valid anymore for data analysis. In such a case, record all animals for this experimental group with the original settings, and create a new experiment afterwards where the recorded videos are analyzed instead of live tracking. In this "from video" experiment, several settings can be used for analysis without losing comparability between animals or even data.

\section{Limitations and future applications}

This method of manipulating behavior with optogenetics in freely moving animals also includes limitations. During the surgery, the proximity of the two implants is restricted. For double implantation, the distance between the two implants must minimally be the width of the apparatus to hold the implant. The apparatus needs to lower the second implant into the burr hole, while the first implants is already fixed. A solution for this might be an angled implantation, where the tips of the glass fiber can be very close while the ceramic ferules above the skull have larger distance $23,55,56,57,62,63$. A disadvantage of an angled implantation is the light spreading. When the fiber tip is slanted instead of from straight above, the stimulated area is different. In case of two target regions in close proximity, the changed position of the light stimulation needs to be considered.

During the behavioral experiment, the construction of the maze might interfere with the optical cable connected to the animal. Some behavioral tests, such as the lightdark box, contain an indoor area64,65, and other mazes contain compartments which the mouse needs to enter. Such experiments cannot be performed with this setup. Alternatively, a wireless system might be an option $22,26,66$. But luckily some mazes, such as the Barnes Maze, can be arranged in such a way, that the mice are able to enter the relevant compartments ${ }^{67}$.

Besides those with closed zones, mazes that are too wide can cause also problems. The larger the area of the maze, the longer the cable has to be to allow the animal to go to every position in the maze. Care has to be taken that the animal is not able to step on the cable or grab it and bite it. A solution for that might be a construction that rolls up the redundant 
cable. A disadvantage is that the drag to unroll the cable is hard for mice. This solution would better suit suited for rats. Another possible option could be to do the light stimulation in advance, instead of during the experiment, of course this is only feasible if a long-term effect due to the light stimulation occurs $^{23}$.

\section{Comparison to existing/alternative methods}

Alternative methods would be chemical or electrical stimulation during behavior 8,18 . Chemical agonists or antagonists are able to activate or silence neurons via specific receptors and can also manipulate single neurotransmitter systems $^{38,68}$. On one hand, the receptor-specificity is quite high for chemicals, because specific agonist or antagonist only activate certain receptors ${ }^{39}$. On the other hand, the specificity for receptor subtypes of the same neurotransmitter group is often insufficient. Most chemicals bind to at least two sub-types with different probabilities ${ }^{69}$. Additionally, chemicals cannot distinguish between neuronal cell types as long as they possess the same receptor types. Beyond this, temporal and spatial resolution is poor for chemical manipulations in comparison to optogenetics. Agonists or antagonists are often administered orally ${ }^{35}$ or via systemic injections ${ }^{57,70}$. If the infusion of the chemical is done directly in the brain tissue, effects appears faster than with oral applications, but still on a slower timescale than with light stimulation. As the administered chemicals diffuse in the brain and are not specific for neuronal types or brain regions, manipulation of specific brain circuitries it not possible.

Electrical stimulation has a higher temporal resolution than chemical stimulation ${ }^{9,14}$. The spread within in neuronal tissue is less than with chemical stimulation and the spatial resolution is better than with chemical stimulation. However, electrical stimulation lacks the possibility to specifically address different neuronal cell types or receptor types, as every neuron in proximity to the electrode will respond to the electrical stimulation.

Alternative methods to the behavior in freely moving mice are for example electrophysiological recordings in brain slices, where single neurons or axons can be modulated with optogenetics and elicited effects can be measured via recording electrodes 6,71 . In vitro experiments offer the possibility to investigate the molecular and cellular basis of optogenetic stimulations but have the limitation that intrinsic connectivity and input from other brain regions is missing. Another option is to use optogenetic in conjunction with multiphoton imaging ${ }^{1,72}$. In this case, mice have their head fixed and can be anesthetized or be awake to solve simple tasks.

To perform a successful optogenetic experiment, a wide range of tools and applications are available nowadays. The selection of optogenetic tools and the behavioral set-up is critical to answer specific research questions. If the right combination of tools and experiments is chosen, optogenetics allows an unprecedented, in-depth investigation of neuronal circuitries with high temporal and spatial resolution. This will help to understand and develop new therapeutic strategies for psychiatric diseases and cognition.

\section{Disclosures}

The authors have nothing to disclose.

\section{Acknowledgments}

Great thanks to Prof Klaus-Armin Narve and Dr. Sandra Goebbels (Max-Plank-Institute of Experimental Medicine, Goettingen, Germany) for kindly providing Nex-Cre mice. Also, we thank our video-team Yunus Dikici and Ruben 
Wiesner for recording and processing of the JoVE video for this article. In addition, great thanks to Kristin Claussen for her voice-over and Kimberly Anne Go for proofreading of the manuscript.

The presented results were obtained at the Ruhr-University in Bochum and the video was recorded at the University of Bremen.

This work was funded by the Deutsche Forschungsgemeinschaft (DFG, German Research Foundation) - Projektnummer 122679504 - SFB 874 and DFG MA 4692/3-2.

\section{References}

1. Chow, B. Y. et al. High-performance genetically targetable optical neural silencing by light-driven proton pumps. Nature Letters. 463, 98-102 (2010).

2. Zhao, S. et al. Cell type-specific channelrhodopsin-2 transgenic mice for optogenetic dissection of neural circuitry function. Nature Methods. 8, 745-752 (2011).

3. Spoida, K., Masseck, O. A., Deneris, E. S., Herlitze, S. $\mathrm{Gq} / 5-\mathrm{HT} 2 \mathrm{c}$ receptor signals activate a local GABAergic inhibitory feedback circuit to modulate serotonergic firing and anxiety in mice. Proceedings of the National Academy of Science of the United States of America. 111, 6479-6484 (2014).

4. Kleinlogel, S. Optogenetic user 's guide to Opto-GPCRs modified GPCRs. Frontiers in Bioscience. 21, 794-805 (2016).

5. Mahn, M., Prigge, M., Ron, S., Levy, R., Yizhar, O. Biophysical constraints of optogenetic inhibition at presynaptic terminals. Nature Neuroscience. 19, 554-556 (2016).
6. Masseck, O. A. et al. Vertebrate Cone Opsins Enable Sustained and Highly Sensitive Rapid Control of Gi/o Signaling in Anxiety Circuitry. Neuron. 81, 1263-1273 (2014).

7. Oh, E., Maejima, T., Liu, C., Deneris, E., Herlitze, S. Substitution of 5-HT 1A Receptor signaling by a light-activated $G$ protein-coupled receptor. Journal of Biological Chemistry. 285, 30825-30836 (2010).

8. Yizhar, O., Fenno, L. E., Davidson, T. J., Mogri, M., Deisseroth, K. Optogenetics in Neural Systems. Neuron Primer. 71, 9-34 (2011).

9. Masseck, O. A. A Guide to Optogenetic Applications, With special Focus on Behavioral and In Vivo Electrophysiological Experiments. in HandboOk of In Vivo Neural Plasticity Techniques - A Systems Neuroscheince Approach to the Neural Basis of Memory and Cognition (ed. Manahan-Vaughan, D.) 557 Academic Press. (2019).

10. Goebbels, S. et al. Genetic Targeting of Principal Neurons in Neocortex and Hippocampus of NEX-Cre Mice. Genesis. 611-621 (2006).

11. Yang, Y. S., Hughes, T. E. Cre Stoplight: A red/green fluorescent reporter of Cre recombinase expression in living cells. Biotechniques. 31, 1036-1041 (2001).

12. Schnütgen, F. et al. A directional strategy for monitoring Cre-mediated recombination at the cellular level in the mouse. Nature Biotechnology. 21, 562-565 (2003).

13. Taniguchi, $H$. et al. A Resource of Cre Driver Lines for Genetic Targeting of GABAergic Neurons in Cerebral Cortex. Neuron. 71, 995-1013 (2011).

14. Deisseroth, K. Optogenetics. Nature Methods. 8, 268-273 (2011). 
15. Palanza, P., Gioiosa, L., Parmigiani, S. Social stress in mice: Gender differences and effects of estrous cycle and social dominance. Physiology and Behavior. 73, 411-420 (2001).

16. Karolewicz, B., Paul, I. A. Group housing of mice increases immobility and antidepressant sensitivity in the forced swim and tail suspension tests. European Journal of Pharmacology. 415, 197-201 (2001).

17. Masseck, O. A., Rubelowski, J. M., Spoida, K., Herlitze, S. Light- and drug-activated G-protein-coupled receptors to control intracellular signalling. Experimental Physiology. 96, 51-56 (2011).

18. Aravanis, A. M. et al. An optical neural interface: in vivo control of rodent motor cortex with integrated fiberoptic and optogenetic technology. Journal of Neural Engineering. 4, (2007).

19. Zhang, F. et al. Multimodal fast optical interrogation of neural circuitry. Nature Article. 446, 633-639 (2007).

20. Owen, S. F., Liu, M. H., Kreitzer, A. C. Thermal constraints on in vivo optogenetic manipulations. Nature Neuroscience. 22, 1061-1065 (2019).

21. Hare, B. D. et al. Optogenetic stimulation of medial prefrontal cortex Drd1 neurons produces rapid and longlasting antidepressant effects. Nature Communication. 10, 1-12 (2019).

22. Allsop, S. A., Vander Weele, C. M., Wichmann, R., Tye, K. M. Optogenetic insights on the relationship between anxiety-related behaviors and social deficits. Frontiers in Behavioral Neuroscience. 8, 1-14 (2014).

23. Fuchikami, M. et al. Optogenetic stimulation of infralimbic PFC reproduces ketamine's rapid and sustained antidepressant actions. Proceedings of the National
Academy of Science of the United States of America. 112, 8106-8111 (2015).

24. Correia, P. A. et al. Transient inhibition and long-term facilitation of locomotion by phasic optogenetic activation of serotonin neurons. Elife. 6, 1-26 (2017).

25. Felix-Ortiz, A. C., Burgos-Robles, A., Bhagat, N. D., Leppla, C. A., Tye, K. M. Bidirectional modulation of anxiety-related and social behaviors by amygdala projections to the medial prefrontal cortex. Neuroscience. 321, 197-209 (2016).

26. Marek, R., Xu, L., Sullivan, R. K. P., Sah, P. Excitatory connections between the prelimbic and infralimbic medial prefrontal cortex show a role for the prelimbic cortex in fear extinction. Nature Brief Communication. (2018).

27. Parfitt, G. M. et al. Bidirectional Control of AnxietyRelated Behaviors in Mice: Role of Inputs Arising from the Ventral Hippocampus to the Lateral Septum and Medial Prefrontal Cortex. Neuropsychopharmacology. 42, 1715-1728 (2017).

28. Bandelow, B., Michaelis, S. Epidemiology of anxiety disorders in the 21st century. Dialogues in Clinical Neuroscience. 17, 327-335 (2015).

29. Kessler, R. C. et al. Lifetime Prevalence and Age-ofOnset Distributions of DSM-IV Disorders in the National Comorbidity Survey Replication. Archives of General Psychiatry. 62, 593-602 (2005).

30. Kessler, R. C., Petukhova, M., Sampson, N. A., Zaslavsky, A. M., Wittchen, H.U. Twelve-month and lifetime prevalence and lifetime morbid risk of anxiety and mood disorders in the United States. International Journal of Methods Psychiatric Research. 21, 169-184 (2014). 
31. Andlin-Sobocki, P., Wittchen, H. U. Cost of anxiety disorders in Europe. European Journal of Neurology. 12, 39-44 (2005).

32. Forster, G. L., Novick, A. M., Scholl, J. L., Wall, M. J. The Role of the Amygdala in Anxiety Disorders. Intech. 61-102 (2012).

33. Liberzon, I. Neural circuits in anxiety and stress disorders: a focused review. Therapeutics and Clinical Risk Management. 11, 115-126 (2015).

34. Sylvers, P., Lilienfeld, S. O., LaPrairie, J. L. Differences between trait fear and trait anxiety: Implications for psychopathology. Clinical Psychology Review. 31, 122-137 (2011).

35. Daws, L. C., Koek, W., Mitchell, N. C. Revisiting Serotonin Reuptake Inhibitors and the Therapeutic Potential of 'Uptake-2' in Psychiatric Disorders. ACS Chemical Neuroscience. 4, 16-21 (2013).

36. Felix-Ortiz, A. C. et al. BLA to vHPC inputs modulate anxiety-related behaviors. Neuron Report. 79, 658-664 (2013).

37. Padilla-Coreano, N. et al. Direct Ventral HippocampalPrefrontal Input Is Required for Anxiety-Related Neural Activity and Behavior. Neuron Article. 89, 857-866 (2016).

38. Lisboa, S. F., Stecchini, M. F., Corrêa, F. M. A., Guimarães, F. S., Resstel, L. B. M. Different role of the ventral medial prefrontal cortex on modulation of innate and associative learned fear. Neuroscience. 171, 760-768 (2010).

39. Bi, L.L. et al. Enhanced excitability in the infralimbic cortex produces anxiety-like behaviors. Neuropharmacology. 72, 148-156 (2013).
40. Yizhar, O. et al. Neocortical excitation/inhibition balance in information processing and social dysfunction. Nature Article. 477, 171-178 (2011).

41. Goebbels, S. et al. Genetic Targeting of Principal Neurons in Neocortex and Hippocampus of NEX-Cre Mice. Genesis. 44, 611-621 (2006).

42. Rubenstein, J. L. R., Merzenich, M. M. Model of autism: increased ratio of excitation/ inhibition in key neural systems. Genes, Brain and Behavior. 2, 255-267 (2003).

43. Berg, L., Eckardt, J., A., M. O. Enhanced activity of pyramidal neurons in the infralimbic cortex drives anxiety behavior. PLoS One. 14, 1-19 (2019).

44. Meunier, C. N. J., Amar, M., Lanfumey, L., Hamon, M., Fossier, P. 5-HT1A receptors direct the orientation of plasticity in layer 5 pyramidal neurons of the mouse prefrontal cortex. Neuropharmacology. 71, 37-45 (2013).

45. Paxinos, G., Franklin, K. B. J., Paxinos, G and Franklin, K. B. J., Paxinos, G., Franklin, K. B. J. Mouse Brain in Stereotaxic Coordinates. Academic Press volume 2nd (2004).

46. Gore, B. B., Soden, M. E., Zweifel, L. S. Manipulating gene expression in projection-specific neuronal populations using combinatorial viral approaches. Current Protocols in Neuroscience. 435, 1-6 (2014).

47. Stujenske, J. M., Spellman, T., Gordon, J. A. Modeling the Spatiotemporal Dynamics of Light and Heat Propagation for InVivo Optogenetics. Cell Report. 12, 525-534 (2015).

48. Berg, L. Imbalance of excitation and inhibition within the prefrontal cortex supports anxiety behavior. Doctoral dissertation. Ruhr-University Bochum. (2019). 
49. Boyden, E. S. A history of optogenetics: The development of tools for controlling brain circuits with light. F1000 Biology Reports. 3, 1-12 (2011).

50. Airan, R. D., Thompson, K. R., Fenno, L. E., Bernstein, H., Deisseroth, K. Temporally precise in vivo control of intracellular signalling. Nature. 458, 1025-1029 (2009).

51. Sparta, D. R. et al. Construction of implantable optical fibers for long-term optogenetic manipulation of neural circuits. Nature Protocol. 7, 12-23 (2012).

52. Covington, H. E. et al. Antidepressant Effect of Optogenetic Stimulation of the Medial Prefrontal Cortex. Journal of Neuroscience. 30, 16082-16090 (2010).

53. Lepicard, E. M., Joubert, C., Hagneau, I., PerezDiaz, F., Chapouthier, G. Differences in anxiety-related behavior and response to diazepam in BALB/cByJ and C57BL/6J strains of mice. Pharmacology, Biochemistry and Behavior. 67, 739-748 (2000).

54. Schmidt, M. V., Müller, M. B. Animal models of anxiety. Elsevier. 3, 369-374 (2006).

55. Cho, J. H., Deisseroth, K., Bolshakov, V. Y. Synaptic Encoding of Fear Extinction in mPFC-amygdala Circuits. Neuron Article. 80, 1491-1507 (2013).

56. Adhikari, A. et al. Basomedial amygdala mediates topdown control of anxiety and fear. Nature. 527, 179-185 (2015).

57. Suzuki, S. et al. The infralimbic and prelimbic medial prefrontal cortices have differential functions in the expression of anxiety-like behaviors in mice. Behavioural Brain Research. 304, 120-124 (2016).

58. Carola, V., D'Olimpio, F., Brunamonti, E., Mangia, F., Renzi, P. Evaluation of the elevated plus-maze and openfield tests for the assessment of anxiety-related behaviour in inbred mice. Behavioural Brain Research. 134, 49-57 (2002).

59. Prut, L., Belzung, C. The open field as a paradigm to measure the effects of drugs on anxiety-like behaviors: A review. European Journal of Pharmacology. 463, 3-33 (2003).

60. Tye, K. M. et al. Amygdala circuitry mediating reversible and bidirectional control of anxiety. Nature Letter. 471, 358-362 (2011).

61. Bouwknecht, J. A. et al. Differential effects of exposure to low-light or high-light open-field on anxietyrelated behaviors: Relationship to c-Fos expression in serotonergic and non-serotonergic neurons in the dorsal raphe nucleus. Brain Research Bulletin. 72, 32-43 (2007).

62. Overstreet, D. H., Knapp, D. J., Angel, R. A., Navarro, M., Breese, G. R. Reduction in repeated ethanol-withdrawalinduced anxiety-like behavior by site-selective injections of 5-HT1A and 5-HT2C ligands. Psychopharmacology (Berlin). 187, 1-12 (2006).

63. Takahashi, A. et al. Glutamate Input in the Dorsal Raphe Nucleus As a Determinant of Escalated Aggression in Male Mice. Journal of Neuroscience. 35, 6452-63 (2015).

64. Klemenhagen, K. C., Gordon, J. A., David, D. J., Hen, R., Gross, C. T. Increased Fear Response to Contextual Cues in Mice Lacking the 5-HT1A Receptor. Neuropsychopharmacology. 31, 101-111 (2006).

65. Ramos, A. Animal models of anxiety: do I need multiple tests? Trends in Pharmacological Science. 29, 493-498 (2008).

66. Isosaka, T. et al. Htr2a-Expressing Cells in the Central Amygdala Control the Hierarchy between Innate and Learned Fear. Cell. 163, 1153-1164 (2015). 
67. Regev, L., Goshen, I. Employing Optogenetics in Memory Research. in Optogenetics: A Roadmap. 219-256 (2017).

68. Shah, A. A., Sjovold, T., Treit, D. Inactivation of the medial prefrontal cortex with the GABA A receptor agonist muscimol increases open-arm activity in the elevated plus-maze and attenuates shock-probe burying in rats. Brain Research. 1028, 112-115 (2004).

69. Knight, A. R. et al. Pharmacological characterisation of the agonist radioligand binding site of $5-\mathrm{HT} 2 \mathrm{~A}, 5-\mathrm{HT} 2 \mathrm{~B}$ and 5-HT2C receptors. Naunyn-Schmiedebergs Archiv of Pharmacology. 370, 114-123 (2004).

70. Graeff, F. G., Viana, M. B., Mora, P. O. Dual Role of 5-HT in Defense and Anxiety. Neuroscience and Biobehavioral Reviews. 21, 791-799 (1997).

71. Cheriyan, J., Sheets, P. L. Altered Excitability and Local Connectivity of mPFC-PAG Neurons in a Mouse Model of Neuropathic Pain. Journal of Neuroscience. 38, 4829-4839 (2018).

72. Boyden, E. S., Zhang, F., Bamberg, E., Nagel, G., Deisseroth, K. Millisecond-timescale, genetically targeted optical control of neural activity. Nature Neuroscience. 8, 1263-1268 (2005). 